\title{
On a Freshwater Gymnodinium sp. with Unusual Nuclear Organization
}

\author{
B. R. Chaudhary and Y. S. R. K. Sarma \\ Laboratory of Algal Cytology and Cytogenetics, Department of Botany, \\ Banaras Hindu University, Varanasi-221005, India
}

Received April 5, 1978

The pioneering work of Dodge $(1960,1963)$ on the nuclear cytology of Dinophyceae, a unique group of algae under 'Mesocaryota' (Dodge 1965), has provided impetus to cytologists all over the world because of an interesting nuclear organisation and nuclear events in the group. The researches carried out in the past two decades have added much to our understanding of this intermediate group. However, our knowledge on nutritional, physiological, cytochemical and biochemical aspects, particularly of freshwater forms is very limited which may necessarily be attributed to the difficulties in the cultivation of these algae under controlled culture conditions. While scanning karyologically suitable dinoflagellate materials for the purposes of cytochemical studies, the authors came across an interesting nucleus in an Indian freshwater dinophyceaen taxon which is being dealt with here in the present communication.

\section{Material and methods}

The material was collected, in the months of Jan.-Feb. 1976, from the experimental cement tanks built in the new Botanical Garden situated in the Banaras University. The algal flagellate in its morphological features resembles Gymnodinium and hence is referred here to as Gymnodinium sp. NBG 76. The alga showed its consistent presence throughout the year, though with variable growth causing golden brown puff patches during its luxuriant growth. It was also found sometimes growing with other phytoplankton components. Several times the phytoplankton was found to be made up of this dinoflagellate exclusively.

The cultivation of the alga was attempted under artificial culture conditions in a thermostatically controlled $\left(22 \pm 1^{\circ} \mathrm{C}\right)$ and fluorescent-illuminated (1400-2000 lux) chamber in a variety of media such as soil-water biphasic medium (Pringsheim 1946), modified Chu No. 10 medium (Chu 1942), Godward's medium (Godward 1942), Carefoot 1336 medium (Carefoot 1968) and von Stosch medium (von Stosch 1973). But unfortunately, the material did not grow luxuriantly in any of the above mentioned media. However, a little success was achieved with Carefoot 1336 medium and von Stosch medium, the latter two proving better. The present study was accomplished mainly on the material collected from nature and in culture in the latter media. The material was fixed in 1:3 acetic-alcohol mixture and stained with aceto-carmine for squash preparations during cytological studies. 


\section{Observations}

Morphological: The alga which is unicellular possesses the morphology of a typical dinoflagellate (Fig. 1). The cells which are more or less spherical range from 25.7-51.4 $\mu \mathrm{m}$ in diameter. The cell is covered by a thin and delicate theca. Transverse furrow is represented by wide constrictions at the region of theca, while longitudinal one is invisible. Longitudinal flagellum is single, posteriorly inserted but without any constriction at the base of its origin. The shape and size of cones (epicone and hypocone) are not distinctly variable. Chromatophores are numerous, round to oval, radially arranged and yellow-brown in colour. Stigma is not visible. Generally the nucleus is more or less spherical and is located towards epicone, sometimes very close to theca of the cone.

Mitosis: The interphase nuclei located in epicone are usually subspherical in shape and measure $8.5-14.3 \mu \mathrm{m}$ in diameter. The nucleus possesses a distinct nuclear membrane which encloses numerous small stained bodies in the nucleoplasm. In favourable preparations single darkly stained round nucleolus measuring 2.1-2.8 $\mu \mathrm{m}$ across can easily be seen in most of the nuclei. The chromosomes in interphase appear as fine dots or short rods (Fig. 2). They are too numerous to be counted for their exact numbers, but rough estimates indicate the presence of $514 \pm 10$ chromosomes (Fig. 2) in the nucleus. The advanced stages, metaphase (Fig. 3) and anaphase (Fig. 4) are easily distinguishable and often appear to be clumped masses of tiny chromosomes. The individuality of some of the chromosomes, however, can easily be made out in favourable preparations. With the present material nothing can be said regarding the nature of the chromosomes except that in shape they are small, dot-like or short rod-like which show uniform condensation throughout the nuclear cycle.

\section{Discussion}

On the basis of the light microscopic studies, the present taxon has been provisionally referred to the genus Gymnodinium as the theca of the organism was very delicate (apparent after disintegration of cells, sometimes even by the weight of the coverslip when the latter was not carefully placed) and devoid of any plate-like structures on the firm periplast left after expulsion of the cellular contents.

Taking all the characters into consideration, the overall conclusion arrived at here pertains to the resemblances at large between the present taxon and Gymnodinium inversum Nygaard (Hubber-Pestalozzi 1968). However, dimensions more appropriately diameters $(25.7-51.4 \mu \mathrm{m})$ of the cells of the former material are far greater than that of the dimension of the latter $(27-36 \mu \mathrm{m} \times 22-29 \mu \mathrm{m})$ which, however, stands close to another variety of the same species i.e., $G$. inversum var. elongatum Nygaard (39-50 $\mu \mathrm{m} \times 22.5-34 \mu \mathrm{m})$. The main difference between the two taxa lies in the longitudinal sulcus which is clearly observable in both the varieties of $G$. inversum, while it is invisible in the present taxon of Gymnodinium.

Although the present material designated here to as Gymnodinium sp. NBG 76 
does not resemble morphologically with the provisionally referred Gymnodinium sp. OBG 70 (Sarma and Shyam 1974), the cytological descriptions of the former taxon largely agree with that of the latter (Sarma and Shyam 1971, Shyam and Sarma 1975). The general appearance of the stained interphase nuclei of the

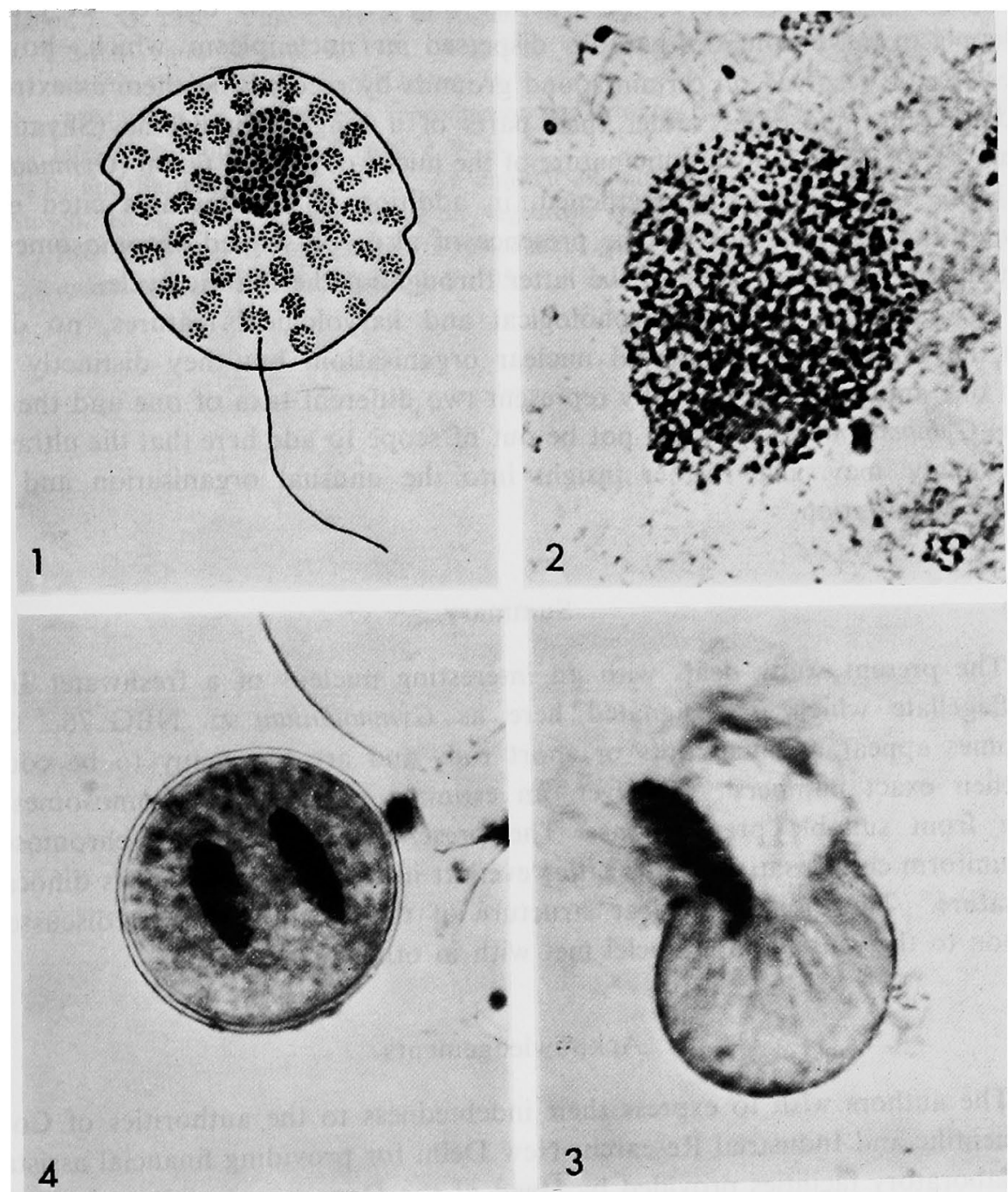

Figs. 1-4. Gymnodinium sp. NBG 76. 1, camera lucida drawing of the organism in ventral view. c. $\times 1150$. 2, squashed interphase nucleus showing numerous dot- or short rod-like chromosomes. c. $\times 3000$. 3 and 4 , metaphase and anaphase plates, respectively with clumped mass of tiny chromosomes. c. $\times 640$.

two taxa is same, but while a count of 600 chromosomes was made in Gymnodinium sp. OBG 70, the number of chromosomes in Gymnodinium sp. NBG 76 was estimated to be $514 \pm 10$. A nucleolus or structure equivalent to it could not be seen even in most favourable preparations of the former taxon, while single darkly stained round nucleolus measuring $2.1-2.8 \mu \mathrm{m}$ in diam. was observed in majority of the 
interphase nuclei of the latter one. Further, the chromosomes in interphase appeared as small dots whose individuality could not be made at metaphase and anaphase in the former, whereas besides fine dots, short rods were also recorded and at the same time the individual identity of the chromosomes was also noticed in the latter taxon. The doubts with the former material were voiced as to the nature of stained dot-like bodies whether they represented descrete or granular nucleoplasm of chromatin granule dispersed in nucleoplasm which, however, were later on resolved on certain sound grounds by recognising them as extremely condensed chromosomes rather than parts of a long chromosome (Shyam and Sarma 1975). The dinocaryotic nature of the nuclei of present taxon (Gymnodinium sp. NBG 76) is further strengthened, in addition to the features cited earlier (Shyam and Sarma 1975), by the presence of also rod-shaped chromosomes and the uniform condensed state of the latter throughout the mitotic cycle.

On summing up the morphological and karyological features, no doubt, both the materials show unusual nuclear organisation, but they distinctly differ from one another and eventually represent two different taxa of one and the same genus Gymnodinium. It would not be out of scope to add here that the ultrastructural study may give further insight into the unusual organisation and their specific delimitation.

\section{Summary}

The present study deals with an interesting nucleus of a freshwater Indian dinoflagellate which is designated here as Gymnodinium sp. NBG 76. Chromosomes appear as small dots or short rods and are too many to be counted for their exact numbers. However, an estimate of $514 \pm 10$ chromosomes was made from suitable preparations. The presence of rod-shaped chromosomes and uniform condensation of the latter even at interphase reflect on its dinocaryotic nature. The unusual nuclear structure of this taxon has been discussed in relation to the dinokaryotic nuclei met with in other taxa.

\section{Acknowledgements}

The authors wish to express their indebtedness to the authorities of Council of Scientific and Industrial Research, New Delhi for providing financial assistance. The laboratory facilities provided by Head of the Department of Botany, Banaras Hindu University is also gratefully acknowledged.

\section{References}

Carefoot, J. R. 1968. Culture and heterotrophy of the freshwater dinoflagellate, Peridinium cinctum fa. ovoplanum Lindeman. J. Phycol, 4: 129.

Chu, S. P. 1942. The influence of the mineral composition of the medium on the growth of planktonic algae I. Methods and culture media. J. Ecology 30: 284.

Dodge, J. D. 1960. Nuclei, nuclear division and taxonomy in the Dinophyceae. Brit. Phycol. Bull. 2: 14 . 
- 1963. The nucleus and nuclear division in the Dinophyceae. Arch. Protistenk. 106: 442.

- 1965. Chromosome structure in the dinofiagellates and the problem of the mesocaryotic cell. Progr. Protozool. 2: 264.

Godward, M.B.E. 1942. The life-cycle of Stigeoclonium amoenum Kütz. New Phytol. 41: 293. Hubber-Pestalozzi, G. 1968. Thienemann's 'Die Binnengewässer' Bd. XVI/3. Das Phytoplankton des Süsswassers (Cryptophyceae, Chloromonadophyceae, Dinophyceae). E. Schweizerbart' sche Verlagsbuchhandlung, Stuttgart.

Pringsheim, E. G. 1946. Pure Cultures of Algae. Cambridge University Press, London.

Sarma, Y.S.R.K. and Shyam, R. 1971. Cytology of some Indian freshwater Dinophyceae. 1st Ind. Congr. Cytol. Genet. (Abs): 16.

- and - 1974. On the morphology, reproduction and cytology of two new freshwater dinoflagellates from India. Brit. Phycol. J. 9: 21.

Shyam, R. and Sarma, Y.S.R.K. 1975. An unusual dinophycean nucleus. The Nucleus 18: 86. von Stosch, H. A. 1973. Observations on vegetative reproduction and sexual life cycles of two freshwater dinoflagellates, Gymnodinium psudopalustre Schiller and Woloszynskia apiculata sp. nov. Br. Phycol. J. 8: 105. 